\title{
Mechanisms and Functions within a National Innovation System
}

\author{
Joseph Gogodze*
}

\begin{abstract}
In modern society, the competitive success of countries is increasingly dependent on the effective management of their national innovation system (NIS). Therefore, understanding the mechanisms behind NISs has become essential. After reviewing the current understanding of the NIS concept and the existing measurement models, this study proposes to consider the NIS as an intangible (underlying) asset of a specific kind and identifies its seven fundamental components, which are extracted with a new measurement model, the Global Innovation Index (GII). This study employs the Structural Equation Modeling (SEM) techniques to analyze the relationships among the components of an NIS. Our results support the existence of a causal link between the constituents of an NIS and provide several perspectives regarding NIS management opportunities. In particular, we find that the efficient management of institutional capital is a key determinant of innovation success for non-high-income countries.
\end{abstract}

Keywords: National Innovation System; Intangible Asset; Structure Equation Model; Global Innovation Index (GII).

Submitted: July $26^{\text {th }} 2016 /$ Approved: November $25^{\text {th }} 2016$

\section{Introduction}

In today's world, fostering innovation is essential to improving economic growth and competitiveness across countries (see e.g. Freeman and Soete, 1997; Gregersen and Johnson, 1997; Klenow and Rodriguez-Clare, 1997; Jaffe and Trajtenberg, 2005; Verspagen, 2005; Fagerberg and Srholec, 2008). However, the literature seems to focus very little on the determinants of innovation and how innovation systems function in different countries. This study attempts to clarify how various countries develop and manage their innovative capacities.

\section{Innovation capability of nations and its measurement}

The OECD/EUROSTAT (2005) provides the following definition of innovation: "An innovation is the implementation of a new or significantly improved product (good or service), or process, a new marketing method, or a new organizational method in business practices, workplace organization or external relations" (p. 46). However, innovation is only a part of a more general process through which new technologies enter the market. According to Schumpeter (1942), this process comprises three stages: invention, innovation, and adoption. Inventions are usually the product of $\mathrm{R} \& \mathrm{D}$ processes and represent only general ideas that may be commercialized in the innovation stage and then distributed on the market in the adoption stage.

The sequence "invention-innovation-adoption" can function efficiently only in the appropriate enabling environment, namely the country's innovation system. According to Gregersen and Johnson (1997), "The main idea of the concept of innovation systems is that the overall innovation performance of an economy depends not only on how specific organizations like firms and research institutes perform but also on how they interact with each other and with the government sector in knowledge production and distribution" (p. 5).
Innovation systems may operate at the regional (sub-national), national, or international level. A National Innovation System (NIS) determines the innovation capability of a country. In other words, an NIS can be seen as a socio-economic system where different actors, such as companies, research and academic organizations, public administrations, professional mediators, and other formal and informal institutions interact. NISs necessarily exploit all available resources in a country, such as human, financial, infrastructural, and institutional resources. Moreover, an NIS requires the generation and dissemination of knowledge, in addition to the utilization of innovation. Finally, the results obtained by NISs can help achieve economic development. An NIS can also be seen as an intangible asset characterized by a set of interacting, underlying (or latent) components able to contribute to a country's economic growth, value creation and wellbeing. At the same time, considering an NIS as an intangible asset requires an appropriate measurement model for it.

The measurement of an NIS (and the corresponding country's innovative capabilities) requires the construction of special instruments to address the complex and multidimensional nature of NISs and adequately reproduce them. Composite indicators are instruments of this type. Various organizations and researchers have developed a number of composite indicators for the measurement of a country's innovative capability, such as the ArCo Index (Archibugi and Coco, 2004 ), Innovation Capability Index (UNCTAD, 2005), TechAchv Index (UNIDO, 2005), Knowledge Economy Index (Chen and Dahlman, 2005), Global Innovation Scoreboard (European Commission, 2007), European Innovation Scoreboard (European Commission, 2008), TechRead Index (WEF, 2009), BCG/NAM Innovation Index (BCG/ NAM, 2009), Economist Intelligence Unit Index (EIU, 2009) and Summary Innovation Index (INNO Metrics, 2011. See also Archibugi and Coco, 2005; Archibugi at al., 2009; Tijssen and Hollanders, 2006; Gogodze, 2013 for other composite indicators and their use).

Institute Techinformi, Georgian Technical University, 47, Kostava St, Tbilisi, Georgia - 0179.

^E-mail: tech@caucasus.net 
The Global Innovation Index (GII, see (INSEAD, 2011-15) for further details) is used in this study. The GII is built on a hierarchical basis and includes two sub-indices composed of seven underlying constructs, named pillars. Each pillar is divided into sub-pillars, and each sub-pillar is the product of individual indicators. This study used the GII data for the period 2011-2015, at the pillar level. Note that we decided to use the GII because it reflects the extensive experience of previous studies and the current understanding of NISs and the mechanisms behind their functioning. In addition, the GII uses well-defined measurement tools, both the primary data and final indicators of the GII are subject to multiple external and internal tests, and the GII is regularly published and contains detailed data on more than 100 countries.

\section{Theoretical assumptions and aims of the study}

On the basis of the above considerations, we introduce the following definition: an NIS is an intangible (underlying) asset (capital) of a country and represents the resources and values of the NIS actors, and the current and potential sources of a country's future economic growth, value creation and wellbeing. Due to the lack of a global theory of NISs, there is currently no clear and full understanding of a country's NIS constituents. However, based on the measurement method introduced by the GII, we can suppose that an NIS contains seven components: Infrastructural capital (INFC), Human and Research capital (H\&RC), Institutional capital (INSC), Market Sophistication capital (MRSC), Business Sophistication capital (BUSC), Knowledge and Technological capital (K\&TC), and Creative Ability capital (CREC). These constituents of NISs should also be considered as intangible assets. We propose to understand them as hidden values of the corresponding NIS actors, and current and potential sources for a country's future development, measured by the respective GII constructs (i.e., GII pillars). We are also assuming that the abovementioned components of NISs form an interaction network, which defines the NIS functioning mechanism and its effectiveness. The aim of the present study is to investigate the channels through which different components of NISs interact with each other.

Figure 1. Conceptual model of NIS sub-systems interactions

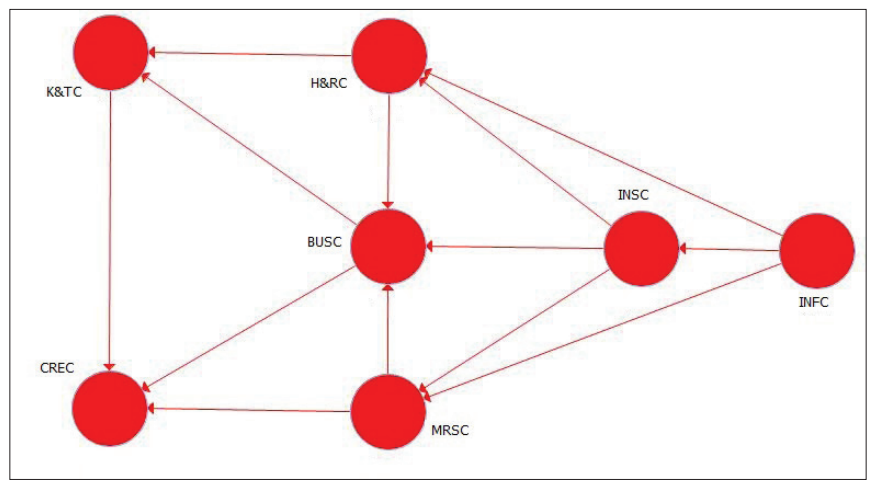

This study develops and explores a conceptual framework for the relationships among NIS components (see Figure 1), and with the hypotheses described below. Specifically, we are assuming that Infrastructural capital (INFC) directly and positively affects Human and Research capital (H\&RC), Market Sophistication capital (MRSC), and Institutional capital (INSC). Consequently, we introduce the following hypotheses:

$$
\begin{aligned}
& \text { H1a: INFC positively affects H\&RC. } \\
& \text { H1b: INFC positively affects MRSC. } \\
& \text { H1c: INFC positively affects INSC. }
\end{aligned}
$$

On the other hand, Institutional capital (INSC) has a direct and positive impact on Human and Research capital (H\&RC), Market Sophistication capital (MRSC), and Business Sophistication capital (BUSC). Accordingly, we introduce the following hypotheses:

\section{H2a INSC positively affects H\&RC.}

H2b: INSC positively affects MRSC.

H2c: INSC positively affects BUSC.

Human and research capital (H\&RC) has a direct and positive impact on Business Sophistication capital (BUSC) and Knowledge and Technological capital (K\&TC). At the same time, Market Sophistication capital (MRSC) has a direct and positive impact on Business Sophistication capital (BUSC) and Creative Ability capital (CREC). Moreover, Business Sophistication capital (BUSC) directly and positively affects Knowledge and Technological capital (K\&TC) and Creative Ability capital (CREC). Finally, Knowledge and Technological capital (K\&TC) has a direct and positive impact on Creative Ability capital (CREC). Accordingly, we introduce the following hypotheses:

H3a: H\&RC positively affects BUSC.

H3b: H\&RC positively affects K\&TC.

H4a: MRSC positively affects BUSC.

H4b: MRSC positively affects CREC.

H5a: BUSC positively affects CREC.

H5b: BUSC positively affects K\&TC.

H6: K\&TC positively affects CREC.

Both the proposed conceptual framework and research hypotheses have been empirically tested in this study, using GII data. 


\section{Method}

\section{Data}

The GII is built on a hierarchical basis and includes two sub-indices: the Innovation Input Sub-Index, composed by five input indexes (pillars), and the Innovation Output Sub-Index, composed by two output indexes. On the next level, each pillar is divided into sub-pillars, and each sub-pillar is the product of the relevant individual indicators. In this study, we used the values of the GII pillars for the period 20112015. To give an intuition about the GII pillars' underlying concepts, in Table $A 1^{1}$ we present the GII structure up to the sub-pillar level, with the corresponding names, as well as the notations used for them in this study. Details about the composition of individual indicators, data sources, processing techniques and country selection methods can be found in (INSEAD,2011-15) and are briefly discussed below.

The GII is the simple average of the above-mentioned input and output sub-indices. Sub-indices are also the simple average of the underlying pillar scores. We compute ach pillar score as the weighted average of its sub-pillar scores, and we derive each sub-pillar score as the weighted average of its individual indicators. The latter are obtained from various sources (their number and composition changes from year to year, and range between 79 and 84 in the GII, for the period 2011-2015). The GII is audited annually by the European Commission Joint Research Centre, which checks the conceptual and statistical consistency of its structure, and the impact of the crucial modeling assumptions on the GII scores and ranking.

In this study, we also use the World Bank classification of countries by income group, as it is presented in the above-mentioned GII publications. The distribution of the GII countries by income group is shown in Table A2. ${ }^{2}$ Based on the World Bank classification, in this study we use the following categorization: "high-income" countries (corresponding to the World Bank classification for high-income countries) and "non-high-income" countries (which include low-income, lower middle-income, and upper middle-income countries, according to the World Bank classification). The sub-samples of GII countries belonging to the high-income and non-high-income countries are named $\mathrm{H}$-sample and $\mathrm{nH}$-sample, respectively.

\section{Analytical procedures}

We used the Structural Equation Modeling (SEM, henceforth) analysis to assess the direct and indirect relationships among the components of an NIS. The core purpose of the SEM is to explain the pattern of a series of interrelated relationships of dependence within a set of latent (or unobserved) variables, measured using manifest (or observed) variables. There are two different techniques to perform SEM analyses: variance and covariance-based SEM. In this study, the variance-based partial least squares (PLS) method was used to evaluate the proposed theoretical model. This decision takes the following circumstances into account: the PLS method can deal with complex models, with a high number of variables and relationships; it allows working with small sample sizes and makes less strict assumptions about the variable distributions, as PLS is primarily intended for causal-predictive analysis of models not backed by strong theory (see Esposito Vinzi at al., 2010).

The analysis of the structural model proposed in this study involves the identification of causal relationships among latent variables, as presented in Figure 1, and the assessment of the explained variance. We used the SmartPLS software (Ringle at al., 2015) to perform the SEM analysis. We carried out a bootstrap analysis in the SmartPLS framework to assess the significance of different statistical characteristics. No less than 5000 sampling generations were performed using a bootstrapping procedure to obtain the final statistical estimations. To investigate the homogeneity of the various groups of data, we used the SmartPLS procedure for Multi Group Analyses (MGA). We utilized the software $G^{*}$ Power 3.1.9 (Faul at al., 2007) to estimate the minimum sample size ${ }^{3}$. Our estimates showed that the minimum sample size to evaluate the model presented in Figure 1 is 77.

\section{Results}

Table 1 shows the descriptive statistics and correlations for the complete sample, as well as for the $\mathrm{H}$-sample and $\mathrm{nH}$-sample. As expected, Table 1 shows statistically significant differences between the $\mathrm{H}$ and $\mathrm{nH}$ sub-samples. For example,

the mean value of the I11 indicator is 80.23 and 52.95 in the $\mathrm{H}$ and $\mathrm{nH}$ sub-sample, respectively; the correlation between the I12 and I14 indicators is 0.09 in the $\mathrm{nH}$ sub-sample, while the same correlation is 0.57 in the $\mathrm{H}$ sub-sample. Thus, a separate consideration for the two sub-samples seems needed. We return to this point below.

(1) see Annex.

(2) see Annex.

(3) For the estimation, we used the following parameters: the test power $\mathrm{P}=0.8$, the Cohen's indicator $\mathrm{f}^{2}=0.15$, the significance level $\alpha=0.05$, and the maximum number of predictors $\mathrm{N}=3$ (see Figure 1), under the G*Power 3.1.9 options: "Linear multiple regression: Fixed model, R² deviation from zero," "F test," "A priori: Compute required sample size" (see Faul at al., 2007). 
Table 1. Descriptive statistics and correlations

\begin{tabular}{|c|c|c|c|c|c|c|c|c|c|c|}
\hline \multirow{2}{*}{ Variable } & \multirow{2}{*}{ Mean } & \multirow{2}{*}{ Std Dev. } & \multirow{2}{*}{ Std Err. } & \multicolumn{7}{|c|}{ Correlation Matrix } \\
\hline & & & & I11 & I12 & I13 & I14 & I15 & I21 & I22 \\
\hline \multicolumn{11}{|c|}{ Complete sample $(\mathrm{N}=692)$} \\
\hline I11 & 61.98 & 16.95 & 0.64 & 1.00 & & & & & & \\
\hline I12 & 33.97 & 14.62 & 0.56 & $0.75^{* * *}$ & 1.00 & & & & & \\
\hline I13 & 33.79 & 13.20 & 0.50 & $0.59^{* * *}$ & $0.59^{* * *}$ & 1.00 & & & & \\
\hline I14 & 46.04 & 12.87 & 0.49 & $0.72^{* * *}$ & $0.59^{* * *}$ & $0.55^{* * *}$ & 1.00 & & & \\
\hline I15 & 36.31 & 11.66 & 0.44 & $0.68^{* * *}$ & $0.73^{* * *}$ & $0.53^{* * *}$ & $0.57^{* * *}$ & 1.00 & & \\
\hline I21 & 28.59 & 12.86 & 0.49 & $0.64^{* * *}$ & $0.73^{* * *}$ & $0.56^{* * *}$ & $0.63^{* * *}$ & $0.69^{* * *}$ & 1.00 & \\
\hline I22 & 33.85 & 12.67 & 0.48 & $0.73^{* * * x}$ & $0.67^{* * *}$ & $0.58^{* * *}$ & $0.61^{* * *}$ & $0.63^{* * *}$ & $0.62^{* * *}$ & 1.00 \\
\hline \multicolumn{11}{|c|}{ H-sample $(\mathrm{N}=229)$} \\
\hline I11 & 80.23 & 9.85 & 0.65 & 1.00 & & & & & & \\
\hline I12 & 49.33 & 10.60 & 0.70 & $0.63^{x+* x}$ & 1.00 & & & & & \\
\hline I13 & 44.12 & 14.78 & 0.98 & $0.23^{*+* x}$ & $0.24^{* * *}$ & 1.00 & & & & \\
\hline I14 & 56.82 & 11.98 & 0.79 & $0.66^{* * x}$ & $0.57^{* * *}$ & $0.27^{* * *}$ & 1.00 & & & \\
\hline I15 & 46.78 & 10.73 & 0.71 & $0.65^{* * *}$ & $0.69^{* * *}$ & $0.28^{* * *}$ & $0.56^{* * *}$ & 1.00 & & \\
\hline I21 & 40.17 & 12.63 & 0.83 & $0.55^{* * *}$ & $0.63^{* * *}$ & $0.30^{* * *}$ & $0.58^{* * *}$ & $0.67^{* * *}$ & 1.00 & \\
\hline $\mathbf{I 2 2}$ & 45.27 & 10.24 & 0.68 & $0.62^{* * *}$ & $0.47^{* * *}$ & $0.32^{* * *}$ & $0.50^{* * *}$ & $0.50^{* * *}$ & $0.48^{* * *}$ & 1.00 \\
\hline \multicolumn{11}{|c|}{ nH-sample $(\mathrm{N}=463)$} \\
\hline I11 & 52.95 & 11.62 & 0.54 & 1.00 & & & & & & \\
\hline I12 & 26.37 & 9.46 & 0.44 & $0.33^{* * *}$ & 1.00 & & & & & \\
\hline I13 & 28.68 & 8.58 & 0.40 & $0.40^{* * *}$ & $0.40^{* * *}$ & 1.00 & & & & \\
\hline I14 & 40.71 & 9.52 & 0.44 & $0.46^{* * *}$ & $0.09^{*}$ & $0.40^{* * *}$ & 1.00 & & & \\
\hline I15 & 31.14 & 8.08 & 0.38 & $0.26^{* * *}$ & $0.38^{* * *}$ & $0.28^{* * *}$ & $0.12^{* *}$ & 1.00 & & \\
\hline I21 & 22.87 & 8.32 & 0.39 & $0.18^{* * *}$ & $0.40^{* * *}$ & $0.36^{* * *}$ & $0.26^{* * *}$ & $0.31^{* * *}$ & 1.00 & \\
\hline I22 & 28.20 & 9.57 & 0.44 & $0.44^{* * *}$ & $0.32^{* * *}$ & $0.42^{* * *}$ & $0.30^{* * *}$ & $0.32^{* * *}$ & $0.28^{* * *}$ & 1.00 \\
\hline
\end{tabular}

Notes: notation for GII pillars given in Table A1, Annex. ${ }^{* * *}-\mathrm{p}<0.01,{ }^{* *}-\mathrm{p}<0.05,{ }^{*}-\mathrm{p}<0.1$.

Table 2 reports the results of the standardized path coefficients (or $\beta$ coefficients) estimation for the proposed model. All path coefficients are statistically significant at the $1 \%$ level in the complete sample. The phase MRSC $\rightarrow$ BUSC is not significant, and all other phases are statistically significant at the $1 \%$ level in the $\mathrm{nH}$ sub-sample. In the $\mathrm{H}$ sub-sample, the phase MRSC $\rightarrow$ BUSC is statistically significant only at the $10 \%$ level, while the phases INFC $\rightarrow \mathrm{H} \& \mathrm{RC}$, INFC $\rightarrow$ MRSC, and K\&TC $\rightarrow$ CREC are statistically significant at the 5\% level, and all other phases are statistically significant at the $1 \%$ level.

Table 2. Estimation of the standardized path coefficients

\begin{tabular}{|c|c|c|c|c|c|c|c|c|c|}
\hline \multirow{2}{*}{ Phase } & \multicolumn{3}{|c|}{ Complete sample } & \multicolumn{3}{|c|}{ H-sample } & \multicolumn{3}{|c|}{ nH-sample } \\
\hline & $\beta$ & STDEV & $\mathrm{T}$ & $\beta$ & STDEV & $\mathrm{T}$ & $\beta$ & STDEV & $\mathrm{T}$ \\
\hline BUSC $\rightarrow$ CREC & 0.321 & 0.046 & $6.996^{* * *}$ & 0.244 & 0.086 & $2.823^{* * * *}$ & 0.246 & 0.052 & $4.698^{* * *+}$ \\
\hline $\mathrm{H} \& \mathrm{RC} \rightarrow \mathrm{BUSC}$ & 0.504 & 0.038 & $13.263^{* * *}$ & 0.445 & 0.062 & $7.168^{* * * x}$ & 0.333 & 0.047 & $7.108^{* *+x}$ \\
\hline $\mathrm{H} \& \mathrm{RC} \rightarrow \mathrm{K} \& \mathrm{TC}$ & 0.480 & 0.037 & $13.040^{* * *}$ & 0.315 & 0.065 & $4.844^{* * *}$ & 0.337 & 0.042 & $8.077^{* * *+}$ \\
\hline INFC $\rightarrow \mathrm{H} \& \mathrm{RC}$ & 0.227 & 0.039 & $5.828^{* * *}$ & 0.107 & 0.051 & $2.097^{* *}$ & 0.321 & 0.043 & $7.409^{*+*+}$ \\
\hline INFC $\rightarrow$ MRSC & 0.191 & 0.038 & $5.038^{* * *}$ & 0.128 & 0.053 & $2.422^{* *}$ & 0.260 & 0.042 & $6.237^{* *+x}$ \\
\hline INSC $\rightarrow$ BUSC & 0.214 & 0.046 & $4.616^{* * *}$ & 0.305 & 0.063 & $4.832^{* * *}$ & 0.135 & 0.051 & $2.635^{* *+}$ \\
\hline INSC $\rightarrow \mathrm{H} \& \mathrm{RC}$ & 0.613 & 0.037 & $16.745^{* * *}$ & 0.601 & 0.045 & $13.435^{* * *}$ & 0.203 & 0.047 & $4.286^{* *+}$ \\
\hline INSC $\rightarrow$ MRSC & 0.609 & 0.033 & $18.536^{* * *}$ & 0.631 & 0.035 & $18.214^{* * *}$ & 0.357 & 0.041 & $8.623^{* *+4}$ \\
\hline $\mathrm{K} \& \mathrm{TC} \rightarrow \mathrm{CREC}$ & 0.212 & 0.042 & $5.101^{* * *}$ & 0.159 & 0.076 & $2.100^{* *}$ & 0.145 & 0.045 & $3.221^{* * x}$ \\
\hline
\end{tabular}

Note: ${ }^{* * *}$-p $<0.01,{ }^{* *}-\mathrm{p}<0.05,{ }^{*}-\mathrm{p}<0.1$ 
The explained variance (or Pearson's coefficient, $\mathrm{R}^{2}$ ) of the dependent variable is a key indicator of the quality of a model. Table 3 reports our estimates of $\mathrm{R}^{2}$ for the dependent variables in the proposed model.

Table 3. The variance explained

\begin{tabular}{|c|c|c|c|c|c|c|c|c|c|}
\hline \multirow{2}{*}{ Variable } & \multicolumn{3}{|c|}{ Complete sample } & \multicolumn{3}{|c|}{ H-sample } & \multicolumn{3}{|c|}{ nH-sample } \\
\hline & $\mathbf{R}^{2}$ & STDEV & $\mathrm{T}$ & $\mathbf{R}^{2}$ & STDEV & $\mathrm{T}$ & $\mathbf{R}^{2}$ & STDEV & $\mathrm{T}$ \\
\hline BUSC & 0.583 & 0.026 & $22.797^{*+* x}$ & 0.564 & 0.042 & $13.411^{* * *}$ & 0.165 & 0.033 & $5.032^{*+x \times}$ \\
\hline H\&RC & 0.590 & 0.025 & $23.206^{* *+}$ & 0.402 & 0.050 & $8.087^{* *+*}$ & 0.197 & 0.035 & $5.689^{2 \times x}$ \\
\hline INSC & 0.343 & 0.044 & $7.866^{* * *}$ & 0.051 & 0.033 & 1.563 & 0.162 & 0.028 & $5.840^{+* x+}$ \\
\hline
\end{tabular}

Note: ${ }^{* *}-\mathrm{p}<0.01,{ }^{* *}-\mathrm{p}<0.05,{ }^{*}-\mathrm{p}<0.1$

For each endogenous variable, the portion of variance explained by the independent variables in the model is sizeable $\left(\mathrm{R}^{2}>0.26\right)^{4}$ in the complete sample. The pattern is the same in the $\mathrm{H}$ sub-sample, except for the INSC variable, whose variance is explained by the IFSC variable only to a small extent $\left(0.02<\mathrm{R}^{2}<0.13\right)$, and is not statistically significant. In the $\mathrm{nH}$ sub-sample, the portion of the variance of the MRSC variable explained by the INSC and IFSC variables is large $\left(\mathrm{R}^{2}>0.26\right)$, but for all other endogenous variables, the portion of variance explained by the model's exogenous variables is rather modest $\left(0.13<\mathrm{R}^{2}<0.26\right)$.
In addition, Table 4 reports the estimates of the magnitude/strength (or Cohen's indicator, $\mathrm{f}^{2}$ ) for each structural path in the proposed model. The size effect assesses the magnitude/strength of the relationship between the variables in the model and shows how much an exogenous latent variable contributes to an endogenous variable's $\mathrm{R}^{2}$. Therefore, size effect is another key indicator of the quality of the proposed approach. Table 4 reports the results for the complete sample: the phase MRSC $\rightarrow$ BUSC has negligible and statistically insignificant strength $\left(\mathrm{f}^{2}<0.02\right)$; the phases BUSC $\rightarrow$ CREC, BUSC $\rightarrow \mathrm{K} \& \mathrm{TC}$, INFC $\rightarrow$ H\&RC, INFC $\rightarrow$ MRSC, INSC $\rightarrow$ BUSC, K\&TC $\rightarrow$ CREC, and MRSC $\rightarrow$ CREC have small strength $\left(0.02<\mathrm{f}^{2}<0.15\right)$; the phases $\mathrm{H} \& \mathrm{RC} \rightarrow \mathrm{BUSC}$ and $\mathrm{H} \& \mathrm{RC} \rightarrow \mathrm{K} \& \mathrm{TC}$ have medium strength $\left(0.15<\mathrm{f}^{2}<0.35\right)$, and the phases INFC $\rightarrow$ INSC, INSC $\rightarrow \mathrm{H} \& \mathrm{RC}$, and INSC $\rightarrow$ MRSC have large strength $\left(0.35<\mathrm{f}^{2}\right)$.

Table 4. Assessment of the effect size

\begin{tabular}{|c|c|c|c|c|c|c|c|c|c|}
\hline \multirow{2}{*}{ Phase } & \multicolumn{3}{|c|}{ Complete sample } & \multicolumn{3}{|c|}{ H-sample } & \multicolumn{3}{|c|}{ nH-sample } \\
\hline & $\mathbf{f}^{2}$ & STDEV & $\mathrm{T}$ & $\mathbf{f}^{2}$ & STDEV & $\mathrm{T}$ & $\mathbf{f}^{2}$ & STDEV & $\mathrm{T}$ \\
\hline BUSC $\rightarrow$ CREC & 0.104 & 0.033 & $3.144^{* * *}$ & 0.045 & 0.036 & 1.248 & 0.067 & 0.031 & $2.189^{*+*}$ \\
\hline $\mathrm{H} \& \mathrm{RC} \rightarrow \mathrm{BUSC}$ & 0.267 & 0.049 & $5.431^{* * *}$ & 0.257 & 0.087 & $2.968^{* * * *}$ & 0.118 & 0.037 & $3.156^{*+1}$ \\
\hline $\mathrm{H} \& \mathrm{RC} \rightarrow \mathrm{K} \& \mathrm{TC}$ & 0.256 & 0.048 & $5.340^{* * * *}$ & 0.102 & 0.047 & $2.192^{* *}$ & 0.120 & 0.033 & $3.608^{*+x}$ \\
\hline INFC $\rightarrow$ H\&RC & 0.082 & 0.027 & $3.082^{* * * *}$ & 0.018 & 0.019 & 0.965 & 0.108 & 0.033 & $3.271^{*+1}$ \\
\hline INFC $\rightarrow$ MRSC & 0.052 & 0.020 & $2.609^{* * *}$ & 0.028 & 0.025 & 1.112 & 0.078 & 0.027 & $2.848^{*+x+1}$ \\
\hline INSC $\rightarrow$ BUSC & 0.036 & 0.016 & $2.230^{* *}$ & 0.101 & 0.041 & $2.465^{* *}$ & 0.015 & 0.012 & 1.256 \\
\hline INSC $\rightarrow$ H\&RC & 0.602 & 0.111 & $5.436^{* * *}$ & 0.574 & 0.133 & $4.304^{* * * *}$ & 0.043 & 0.022 & $1.994^{* *}$ \\
\hline INSC $\rightarrow$ MRSC & 0.535 & 0.089 & $6.030^{* * * *}$ & 0.689 & 0.123 & $5.610^{* * * *}$ & 0.146 & 0.039 & $3.787^{*+1}$ \\
\hline $\mathrm{K} \& \mathrm{TC} \rightarrow \mathrm{CREC}$ & 0.041 & 0.016 & $2.513^{* *}$ & 0.018 & 0.018 & 1.005 & 0.022 & 0.014 & 1.538 \\
\hline
\end{tabular}

Note: ${ }^{* * *}-\mathrm{p}<0.01,{ }^{* *}-\mathrm{p}<0.05,{ }^{*}-\mathrm{p}<0.1$.

(4) In this study, we also used threshold values (or rules of thumb) for the Pearson's coefficient, $\mathrm{R}^{2}$, and Cohen's indicator, $\mathrm{f}^{2}$, usually employed in the standard practice: $\mathrm{R}^{2}<0.02$ (negligible), $0.02<\mathrm{R}^{2}<0.13$ (weak), $0.13<\mathrm{R}^{2}<0.26$ (medium), $0.26<\mathrm{R}^{2}$ (large); $\mathrm{f}^{2}<0.02$ (negligible), $0.02<\mathrm{f}^{2}<0.15$ (small), $0.15<\mathrm{f}^{2}<0.35$ (medium), $0.35<\mathrm{f}^{2}$ (large). 
In the $\mathrm{H}$ sub-sample, we observe that the phases INFC $\rightarrow \mathrm{H} \& \mathrm{RC}$, K\&TC $\rightarrow$ CREC, and MRSC $\rightarrow$ BUSC have negligible and statistically insignificant strength $\left(\mathrm{f}^{2}<0.02\right)$; BUSC $\rightarrow \mathrm{CREC}, \mathrm{H} \& \mathrm{RC} \rightarrow \mathrm{K} \& \mathrm{TC}$, INFC $\rightarrow$ INSC, INFC $\rightarrow$ MRSC, INSC $\rightarrow$ BUSC, and MRSC $\rightarrow$ CREC have medium strength $\left(0.15<\mathrm{f}^{2}<0.35\right)$; the phases $\mathrm{BUSC} \rightarrow \mathrm{CREC}$, INFC $\rightarrow$ INSC, and INFC $\rightarrow$ MRSC also have statistically insignificant strength, and the phases BUSC $\rightarrow$ K\& $\& C$ and $\mathrm{H} \& \mathrm{RC} \rightarrow \mathrm{BUSC}$ have a large strength $\left(0.35<\mathrm{f}^{2}\right)$. In the $\mathrm{nH}$ sub-sample, the phases INSC $\rightarrow$ BUSC and MRSC $\rightarrow$ BUSC have a negligible and statistically insignificant strength $\left(\mathrm{f}^{2}<0.02\right)$; only the phase INFC $\rightarrow$ INSC has large strength $\left(0.35<\mathrm{f}^{2}\right)$; all other phases have a medium strength $\left(0.15<\mathrm{f}^{2}<0.35\right)$, and the phase K\&TC $\rightarrow$ CREC has statistically insignificant strength.

Table 5. Phase differences comparison for sub-samples across time

\begin{tabular}{|c|c|c|c|c|c|c|c|c|c|c|}
\hline \multirow{2}{*}{ Phase } & \multicolumn{5}{|c|}{ H-sample vs. } & \multicolumn{5}{|c|}{ nH-sample vs. } \\
\hline & H_2011 & H_2012 & H_2013 & H_2014 & H_2015 & nH_2011 & nH_2012 & nH_2013 & nH_2014 & nH_2015 \\
\hline BUSC $\rightarrow$ CREC & 0.183 & 0.282 & 0.178 & 0.144 & 0.111 & 0.252 & 0.049 & 0.194 & 0.140 & 0.019 \\
\hline BUSC $\rightarrow$ K\&TC & 0.057 & 0.006 & 0.100 & 0.025 & 0.205 & 0.050 & 0.202 & 0.022 & 0.084 & 0.079 \\
\hline H\&RC $\rightarrow$ BUSC & 0.107 & 0.133 & 0.149 & 0.154 & 0.073 & 0.095 & 0.021 & 0.090 & 0.164 & 0.174 \\
\hline $\mathrm{H} \& \mathrm{RC} \rightarrow \mathrm{K} \& \mathrm{TC}$ & 0.070 & 0.010 & 0.042 & 0.091 & 0.114 & 0.083 & 0.005 & 0.028 & 0.160 & 0.147 \\
\hline INFC $\rightarrow \mathrm{H} \& \mathrm{RC}$ & 0.478 & 0.011 & 0.035 & 0.043 & 0.045 & 0.192 & 0.060 & 0.143 & 0.359 & 0.269 \\
\hline INFC $\rightarrow$ INSC & 0.456 & 0.146 & 0.151 & 0.101 & 0.194 & 0.033 & 0.053 & 0.014 & 0.111 & 0.166 \\
\hline INFC $\rightarrow$ MRSC & 0.362 & 0.082 & 0.056 & 0.040 & 0.056 & 0.042 & 0.103 & 0.054 & $0.172^{*}$ & $0.160^{*}$ \\
\hline INSC $\rightarrow$ BUSC & 0.040 & 0.041 & 0.005 & 0.006 & 0.065 & $0.205^{*}$ & $0.180^{*}$ & 0.065 & 0.270 & 0.217 \\
\hline INSC $\rightarrow$ H\&RC & $0.422^{* * *}$ & 0.055 & 0.061 & 0.049 & 0.069 & 0.124 & 0.005 & 0.021 & $0.209^{*}$ & $0.185^{*}$ \\
\hline INSC $\rightarrow$ MRSC & $0.327^{* *}$ & 0.064 & 0.023 & 0.028 & 0.073 & 0.055 & 0.018 & 0.088 & 0.004 & 0.098 \\
\hline K\&TC $\rightarrow$ CREC & 0.073 & 0.027 & 0.099 & 0.108 & 0.057 & 0.044 & 0.079 & $0.182^{*}$ & 0.135 & 0.163 \\
\hline MRSC $\rightarrow$ BUSC & 0.202 & 0.149 & 0.143 & 0.139 & 0.057 & 0.392 & 0.224 & 0.157 & 0.036 & 0.065 \\
\hline MRSC $\rightarrow$ CREC & 0.090 & 0.366 & 0.104 & 0.191 & 0.087 & 0.139 & 0.159 & 0.001 & 0.167 & 0.106 \\
\hline
\end{tabular}

Note: ${ }^{* *}-\mathrm{p}<0.01,{ }^{* *}-\mathrm{p}<0.05,{ }^{*}-\mathrm{p}<0.1$.

Let us consider now the issue of homogeneity across time, which is one of the fundamental assumptions of this study. To this end, we split the $\mathrm{H}$ and $\mathrm{nH}$ sub-samples into smaller sub-samples, by year, and conduct the relevant comparisons for the phase coefficients for each sub-sample, and for the $\mathrm{H}$ and $\mathrm{nH}$ sub-samples, respectively. Our results are reported in Table 5. Almost all differences in the coefficients are statistically insignificant at the $5 \%$ level. The only exceptions are the differences in the phase INSC $\rightarrow \mathrm{H} \& \mathrm{RC}$ coefficients (significant at the $1 \%$ level) and phase INSC $\rightarrow$ MRSC coefficients (significant at $5 \%$ level) for both the $\mathrm{H}$ sub-sample and the $\mathrm{H}-2011$ sub-sample. This observation allows us to conclude that our assumption of homogeneity over time seems acceptable. To investigate the main features of NIS functioning in both high- and non-high-income countries, we have also compared the path coefficients of the $\mathrm{H}$ and $\mathrm{nH}$ sub-samples. Our results are reported in Table 6.

Table 6. Phase differences comparison for the $\mathrm{H}$ and $\mathrm{nH}$ sub-samples

\begin{tabular}{|c|c|c|c|}
\hline & $\begin{array}{l}\text { Phase } \\
\text { difference }\end{array}$ & $\begin{array}{l}\text { T-value by Parametric } \\
\text { Test }\end{array}$ & $\begin{array}{l}\text { T-value by } \\
\text { Welch-Satterthwait } \\
\text { Test }\end{array}$ \\
\hline BUSC $\rightarrow$ CREC & 0.002 & 0.019 & 0.018 \\
\hline $\mathrm{H} \& \mathrm{RC} \rightarrow \mathrm{BUSC}$ & $0.112^{*}$ & 1.411 & 1.444 \\
\hline $\mathrm{H} \& \mathrm{RC} \rightarrow \mathrm{K} \& \mathrm{TC}$ & 0.022 & 0.296 & 0.288 \\
\hline INFC $\rightarrow$ MRSC & 0.132 & $1.878^{*}$ & $1.957^{*}$ \\
\hline INSC $\rightarrow$ BUSC & $0.170^{* *}$ & $1.991^{* *}$ & $2.073^{* *}$ \\
\hline INSC $\rightarrow \mathrm{H} \& \mathrm{RC}$ & 0.398 & $5.442^{* * *}$ & $6.153^{* * x+}$ \\
\hline INSC $\rightarrow$ MRSC & $0.274^{* * *}$ & $4.280^{* * * *}$ & $5.010^{* * * *}$ \\
\hline
\end{tabular}

Note: ${ }^{* * *}-\mathrm{p}<0.01,{ }^{* *}-\mathrm{p}<0.05,{ }^{*}-\mathrm{p}<0.1$. 
The differences between the path coefficients for the $\mathrm{H}$ and $\mathrm{nH}$ subsamples are statistically significant at the $10 \%$ level for the following phases: BUSC $\rightarrow$ K\&TC, INFC $\rightarrow \mathrm{H} \&$ RC, INFC $\rightarrow$ MRSC, INFC $\rightarrow$ INSC, INFC $\rightarrow$ MRSC, INSC $\rightarrow$ BUSC, INSC $\rightarrow$ H\&RC, and INSC $\rightarrow$ MRSC, (i.e., for 7 over 13 phases). The above-mentioned results suggest that the proposed model of NIS functioning is satisfactory regarding quality, as it relevantly shows the direction and magnitude of the interactions among the components of NISs.

\section{Discussion}

The model developed in this study provides empirical support for the proposed research hypothesis. Moreover, our results showed that the magnitude of the NIS components' interactions considerably depends on the level of economic development in a country. Our results also show that Institutional capital (INSC) is a fundamental constituent of NISs. Although Institutional capital (INSC) does not directly impact a country's innovation output (while other components, such as K\&TC and CREC, do), it has a positive and significant impact on innovation capability through Human capital and Research, and Business and Market Sophistication (measured by the H\&RC, BUSC, and MRSC constituents of NISs). Therefore, the quality of a country's political, regulatory, and business environment indirectly, but fundamentally, affects its innovation capabilities. In particular, the efficient management of a country's Institutional capital helps establish fruitful relationships with the other components of a country's NIS. Institutional capital's direct impact on H\&RC, BUSC, and MRSC is noticeably weaker for non-high-income countries. Therefore, the non-high-income countries should focus on improving their Institutional capital to increase their innovative capabilities.

Institutional capital (INSC) is directly and positively influenced by Infrastructure capital (INFC), but the impact is weaker for high-income countries. The same can be observed for H\&RC and MRSC, which are also directly and positively affected by INFC, probably because infrastructure capital has reached a level of saturation in high-income countries. Hence, non-high-income countries should focus on improvements in infrastructure capital. Human and Research capital (H\&RC) directly and positively affects Business Sophistication and Knowledge and Technology capital (BUSC and K\&TC, respectively). Note also that the impact of Market Sophistication (MRSC) on Business Sophistication (BUSC) is very small, but its impact on Creative Ability (CREC) is significant. Business sophistication (BUSC) has a direct and positive impact on Knowledge and Technology (K\&TC) and Creative Ability (CREC). The effect of Knowledge and Technology capital (K\&TC) on Creative Ability (CREC) is also direct and positive. To summarize, Table 7 presents the assessments of the overall effects of the NIS components' interactions.

Table 7. Total effects of interactions

\begin{tabular}{|c|c|c|c|c|c|c|}
\hline & \multicolumn{3}{|c|}{ H-sample } & \multicolumn{3}{|c|}{ nH-sample } \\
\hline & Mean & STDEV & t-Values & Mean & STDEV & t-Values \\
\hline BUSC $\rightarrow$ CREC & 0.315 & 0.073 & $4.320^{* * *}$ & 0.272 & 0.049 & $5.556^{* * *}$ \\
\hline BUSC $\rightarrow$ K\&TC & 0.448 & 0.063 & $7.080^{* * *}$ & 0.177 & 0.049 & $3.613^{* * *+}$ \\
\hline $\mathrm{H} \& \mathrm{RC} \rightarrow \mathrm{BUSC}$ & 0.445 & 0.062 & $7.176^{* * *}$ & 0.333 & 0.047 & $7.148^{* * *+}$ \\
\hline H\&RC $\rightarrow$ CREC & 0.190 & 0.041 & $4.671^{* * *+}$ & 0.139 & 0.023 & $5.953^{+* x+}$ \\
\hline $\mathrm{H} \& \mathrm{RC} \rightarrow \mathrm{K} \& \mathrm{TC}$ & 0.515 & 0.054 & $9.467^{* * *}$ & 0.395 & 0.037 & $10.694^{* *+}$ \\
\hline INFC $\rightarrow$ BUSC & 0.207 & 0.052 & $3.913^{* * *}$ & 0.201 & 0.030 & $6.670^{* * *+}$ \\
\hline INFC $\rightarrow$ CREC & 0.152 & 0.041 & $3.661^{* * *}$ & 0.170 & 0.024 & $7.046^{* * *}$ \\
\hline INFC $\rightarrow$ H\&RC & 0.245 & 0.064 & $3.812^{* * *}$ & 0.404 & 0.040 & $10.196^{* * *}$ \\
\hline INFC $\rightarrow$ INSC & 0.228 & 0.071 & $3.194^{* * *+}$ & 0.402 & 0.034 & $11.816^{* * *}$ \\
\hline INFC $\rightarrow$ K\&TC & 0.170 & 0.044 & $3.800^{* * *}$ & 0.172 & 0.025 & $6.777^{* * *+}$ \\
\hline INFC $\rightarrow$ MRSC & 0.272 & 0.066 & $4.090^{* * * *}$ & 0.405 & 0.037 & $11.026^{* *+}$ \\
\hline INSC $\rightarrow$ BUSC & 0.635 & 0.036 & $17.533^{* * *}$ & 0.213 & 0.044 & $4.791^{* * *}$ \\
\hline INSC $\rightarrow$ CREC & 0.400 & 0.040 & $10.026^{* * *}$ & 0.152 & 0.023 & $6.473^{* * *+}$ \\
\hline INSC $\rightarrow$ H\&RC & 0.600 & 0.045 & $13.298^{* * *}$ & 0.203 & 0.046 & $4.390^{* * *+}$ \\
\hline INSC $\rightarrow$ K\&TC & 0.475 & 0.037 & $12.747^{*+*}$ & 0.106 & 0.021 & $5.046^{* * *}$ \\
\hline INSC $\rightarrow$ MRSC & 0.630 & 0.036 & $17.615^{* * *}$ & 0.356 & 0.041 & $8.613^{* *+*}$ \\
\hline $\mathrm{K} \& \mathrm{TC} \rightarrow \mathrm{CREC}$ & 0.158 & 0.074 & $2.148^{* *}$ & 0.144 & 0.044 & $3.271^{* * *+}$ \\
\hline MRSC $\rightarrow$ BUSC & 0.099 & 0.061 & $1.673^{*}$ & 0.028 & 0.049 & 0.564 \\
\hline MRSC $\rightarrow$ CREC & 0.298 & 0.066 & 4.558 & 0.241 & 0.046 & $5.246^{*}$ \\
\hline MRSC $\rightarrow$ K\&TC & 0.044 & 0.028 & 1.610 & 0.006 & 0.010 & 0.515 \\
\hline
\end{tabular}

Note: ${ }^{* *}-\mathrm{p}<0.01,{ }^{* *}-\mathrm{p}<0.05,{ }^{*}-\mathrm{p}<0.1$ 
This study proposes to consider the NIS as an intangible asset of a particular kind and identifies its seven fundamental constituents. These components are extracted through a modern NIS measurement model, the Global Innovation Index (GII), and are named conventionally: Infrastructural capital, Human and Research capital, Institutional capital, Market Sophistication capital, Business Sophistication capital, Knowledge and Technological capital, and Creative Ability capital. Based on empirical data and applying the Structural Equation Model (SEM) analysis techniques, this study establishes the existence of a causal relation between the proposed NIS components and the assessed magnitude of their interactions.

The results of this study on the NIS functioning can serve as a basis for several practical applications, potentially leading to new management capabilities of NISs. We found that a country's ability to efficiently manage its own Institutional capital's quality and its interaction with other NIS components is a key determinant of successful innovation. The transformation of infrastructural capacities into effectively functioning human, research, market, and business constituents of NISs is entirely determined by Institutional capital and, as showed in this study, is significantly affected by the innovation output of a country (i.e., Knowledge, Technological and Creativity Ability). The proposed model, as well as our parameter estimates, may enable researchers and practitioners to rank the relative importance of different managing practices to enhance NIS effectiveness.

The results of this study also highlight the following areas of future research: an additional analysis for a clear understanding of NIS as a transformation mechanism for economic development; classification issues and detailed analyses of NIS functioning, considering other factors (e.g., cultural characteristics, geographical location, stocks of mineral resources and/or others); modeling the spatiotemporal evolution of NIS distribution.

\section{References}

Archibugi, D., Coco, A., 2004. New indicator of technological capabilities for developed and developing countries (ArCo). World Development 32(4), 629-654. doi:10.1016/j.worlddev.2003.10.008

Archibugi, D., Coco, A., 2005. Measuring technological capabilities at the country level: A survey and a menu for choice. Research Policy 34, 175-194. doi:10.1016/j.respol.2004.12.002

Archibugi, D., Denni, M., Filippetti, A., 2009. The technological capabilities of nations: The state of the art of synthetic indicators. Technological Forecasting and Social Change 76, 917-931. doi:10.1016/j. techfore.2009.01.002

BCG/NAM, 2009 (2016, May 11). The innovation imperative in manufacturing: How the United States can restore its edge. BCG (Boston Consulting Group / National Association of Manufacturers), Boston. Retrieved from

http://www.bcg.com/documents/file15445.pdf.
Chen, D., Dahlman, C., 2005. The Knowledge Economy, the KAM Methodology and World Bank Operations. World Bank Institute Working Paper No. 37256, World Bank, Washington DC.

Esposito Vinzi, V., Trinchera, L., \& Amato, S. (2010). PLS path modeling: From foundations to recent developments and open issues for model assessment and improvement, in: V. Esposito Vinzi, W.W. Chin, J. Henseler \& H. Wang (Eds), Handbook of Partial Least Squares: Concepts, Methods and Applications. Springer Berlin Heidelberg, Berlin, pp. 47-82.

EIU, 2009 (2016, May 11). A new ranking of the world's most innovative countries. An Economist Intelligence Unit Report Sponsored by Cisco. Retrieved from http://graphics.eiu.com/PDF/Cisco_Innovation_Complete.pdf.)

European Commission, 2007. Global innovation scoreboard 2006. Directorate-General for Enterprise and Industry, Brussels.

European Commission, 2008. European innovation scoreboard 2007. Directorate-General for Enterprise and Industry, Brussels.

Fagerberg, J., Srholec, M., 2008. National innovation systems, capabilities and economic Development. Research Policy 37, 1417-1435.

Faul, F., Erdfelder, E., Lang, A.G., Buchner, A., 2007. G* Power 3: A flexible statistical power analysis program for the social, behavioral, and biomedical sciences. Behavior Research Methods, 39, 175-191.

Freeman, C., Soete, L., (1997). The Economics of Industrial Innovation. Pinter, London.

Gogodze, J., 2013. The composite ECAICI: positioning of Georgia's innovative capacities in the Europe-Central Asia region. International Journal of Scientific \& Technology Research 2 (9), 110-119.

Gregersen, B., Johnson, B., 1997. How do innovations affect economic growth? Some different approaches in economics. Report within the ISE Innovation Systems and European Integration.

INNO Metrics, 2011. Innovation union scoreboard 2010, UNU-MERIT, Maastricht.

INSEAD, 2011-15. Global innovation index 2011, Global innovation index 2012, Global innovation index 2013, Global innovation index 2014, Global innovation index 2015, INSEAD.

Jaffe, A.B., Trajtenberg, M., 2005. Patents, Citations, and Innovations: A Window on the Knowledge Economy. The MIT Press.

Klenow, P., Rodriguez-Clare, A., 1997. The neoclassical revival in growth economics: Has it gone too far? NBER Macroeconomics Annual 12, 73-103.

OECD/EUROSTAT, 2005. The Measurement of Scientific and Technological Activities. Oslo Manual (3rd ed.) OECD. 
Ringle, C.M., Wende, S., Becker, J.M., 2015. Smart PLS 3. Boenningstedt: SmartPLS GmbH; http://www.smartpls.com/.

Schumpeter, J.A., 1942. Capitalism, Socialism, and Democracy. Allen \& Unwin, London.

Tijssen, R., Hollanders, H., 2006. Using science and technology indicators to support knowledge based economies. UNU-MERIT, Policy Brief 11, Maastricht.

UNCTAD, 2005. World investment report. Transnational corporations and the internationalization of R\&D. UNCTAD, Geneva.
UNIDO, 2005. Industrial development report. Capability building for catching-up. Historical, empirical and policy dimensions. UNIDO, Vienna.

Verspagen, B., 2005. Innovation and economic growth, in: Fagerberg, J., Mowery, D. C., Nelson R. R. (Eds.), The Oxford Handbook of Innovation, Oxford University Press, pp. 487-513.

WEF, 2009. The Global Competitiveness Report 2009-2010. World Economic Forum, Geneva.

\section{Annex}

Table A1. GII composition (first 3 levels) and weights

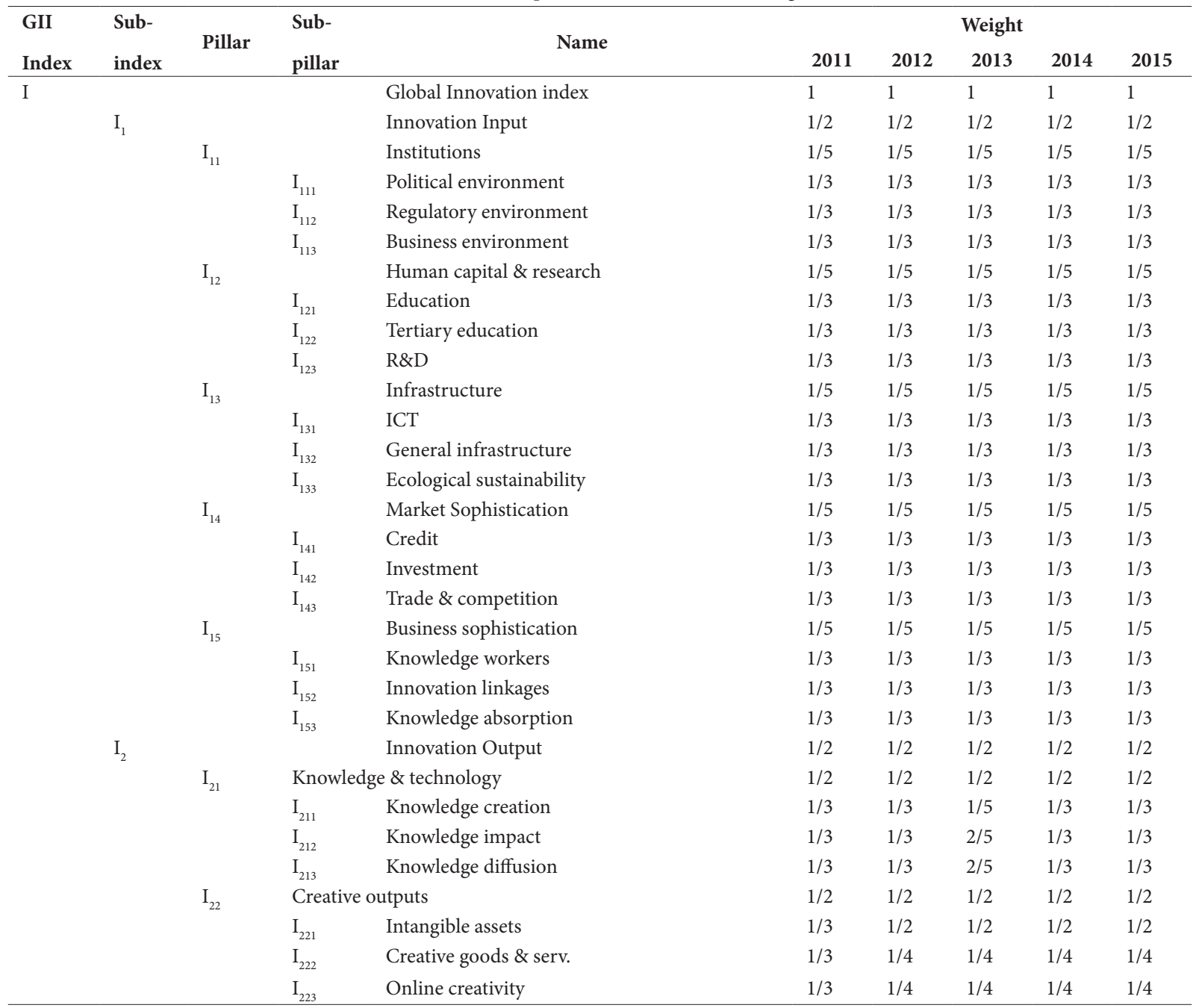

Note: Pillar $\mathrm{I}_{21}$ "Knowledge and technology outputs" and Sub-pillar $\mathrm{I}_{123}$ "Ecological sustainability" were named "Scientific outputs" and "Energy," respectively, in the GII 2011. Sub-pillar $\mathrm{I}_{223}$ "online creativity" was not introduced in the GII 2011. 
Table A2. GII samples by year

\begin{tabular}{llcccc}
\hline \multirow{2}{*}{ Year } & \multicolumn{2}{c}{ Number of countries by income level } & Total \\
\hline $\mathbf{2 0 1 1}$ & H & UM & LM & L & 125 \\
$\mathbf{2 0 1 2}$ & 43 & 35 & 31 & 16 & 141 \\
$\mathbf{2 0 1 3}$ & 44 & 40 & 35 & 22 & 142 \\
$\mathbf{2 0 1 4}$ & 45 & 40 & 36 & 21 & 143 \\
$\mathbf{2 0 1 5}$ & 49 & 38 & 33 & 23 & 141 \\
\hline
\end{tabular}

Note: H- High-income countries; UM- Upper-Middle-income countries; LM- Lower-Middle-income countries; L- Low-income countries. 